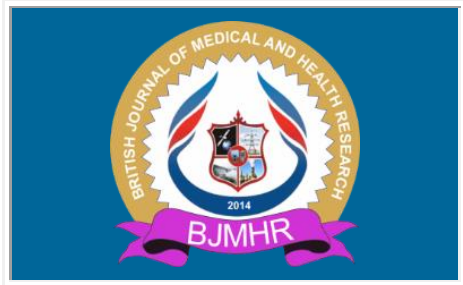

\title{
BJMHR
}

British Journal of Medical and Health Research

Journal home page: www.bjmhr.com

\section{Pyrexia of Unknown Origin and Skeletal Tuberculosis}

\section{Sajani Deepakkumar Kapadia*1, Mrugal Vipul Doshi², Manish R. Shah³, Jitendra Lakhani ${ }^{4}$}

1.Third Year Medicine Resident, Department of General Medicine, Dhiraj Hospital, Gujarat, India

2.Ex Medicine Resident, Department of General Medicine, Dhiraj Hospital, Gujarat, India

3.Associate professor, Department of Orthopaedics, Dhiraj Hospital, Gujarat, India. 4.Professor, Head of Unit and Academic Director, Department of General Medicine, Dhiraj Hospital, Gujarat, India.

\section{ABSTRACT}

Skeletal tuberculosis is one of the common forms of extra pulmonary tuberculosis of which $50 \%$ of the cases are of spinal tuberculosis and the rest are tubercular arthritis or tendinitis or osteomyelitis $^{3-5}$. We report a case of "caries sicca" type of osteoarticular tuberculosis of the shoulder joint, who presented to us primarily for pyrexia. She was investigated at outpatient department as well at the time of previous indoor admissions. She presented to us as her fever persisted despite her admissions twice in multi-specialty hospital. Apart from fever, her relatives noticed right frozen shoulder for which Magnetic Resonance Imaging (MRI) was done which showed changes of arthropathy and synovial effusion. Arthrocentesis was positive for Mycobacterium tuberculosis by Cartridge Based Nucleic Acid Amplification Test. The patient was started on anti-tubercular drugs and physical rehabilitation, upon which she improved. Diagnosis can be missed in such a patient presenting as pyrexia of unknown origin without obvious swelling or sinus at the joint. Extra pulmonary tuberculosis can be one of the common causes of patient presenting with pyrexia of unknown origin; tuberculosis of the shoulder joint is even rarer.

Keywords: Fever, extra pulmonary tuberculosis, shoulder joint, tuberculous arthropathy.

*Corresponding Author Email: sajnikapadia5@gmail.com

Received 05 February 2021, Accepted 18 February 2021

Please cite this article as: Kapadia SD et al., Pyrexia of Unknown Origin and Skeletal

Tuberculosis. British Journal of Medical and Health Research 2021. 


\section{INTRODUCTION}

A true test a physician faces in a day-to-day practice is the diagnosis of patient with prolonged pyrexia. There are varied causes of pyrexia of unknown origin; the variation depends on area, healthcare infrastructure and socio-economic demographics of the patient. Tuberculosis is an important cause of fever of unknown origin ${ }^{1}$. In developing countries, Pyrexia of Unknown Origin (PUO) due to infections remains predominant cause, more specifically extra pulmonary tuberculosis and military tuberculosis ${ }^{2}$.

For the diagnosis of tuberculosis, isolation of the tubercular bacillus and/or histopathological diagnosis from the tissue or body fluid is vital which can be obtained by FNAC or biopsy. However, to obtain the desired tissue is a tedious task. Skeletal tuberculosis (TB) is one of the common forms of extra pulmonary tuberculosis of which $50 \%$ of the cases are of spinal tuberculosis and the rest are tubercular arthritis or tendinitis or osteomyelitis $\mathrm{s}^{3-5}$. The prevalence of tuberculosis of the shoulder joint is very low, which is 0.9 to 1.7 percent of skeletal tuberculosis and can be misdiagnosed as frozen shoulder'. We report a case of "caries sicca" type of osteoarticular tuberculosis of shoulder joint, presenting as pyrexia of unknown origin (PUO).

\section{CASE REPORT:}

A 55-year-old female presented with complaints of intermittent fever with chills and rigors that increased in the evening and was associated with anorexia and vomiting for preceding two months. She did not have any comorbidities before this illness. Patient went to family practitioner and later on, was admitted twice in a multispecialty hospital for fever, where she was investigated and treated. As fever did not subside, she came to us for diagnosis and further management. Patient's relatives noticed restricted and painful movement of right shoulder joint of last 15 days which was labelled by them as "Frozen Shoulder". On asking patient about the same, she volunteered history of difficulty in wearing clothes and raising her hand above the shoulder. There was no past history of pulmonary or extra pulmonary tuberculosis, immunodeficiency state, sexually transmitted diseases or any other infections. She did not have any contact to person having tuberculosis, no other joints were involved nor was there any history of inflammatory arthritis in the past.

On examination she was having temperature of 101 degree Fahrenheit; other vitals were stable with no pallor, lymphadenopathy, and no abnormality in her systemic examination. Examination of her shoulder joint was suggestive of raised temperature with shiny skin over the right shoulder. There was no discharging sinus or abscess, movement of her shoulder joint was painful and restricted in all directions but without any neurovascular deficit.

She was admitted to some other hospital two times before where she underwent a battery of investigations for her fever. These investigations were suggestive of normocytic normochromic 
anaemia with no leucocytosis or leukopenia and no thrombocytopenia as well as no evidence of malaria on Gimesa stained thick/thin smear and rapid dipstick antigen capture assay. Her complete blood picture, urine examination, tests for typhoid fever, Dengue, Leptospira and others were not contributing in the diagnosis of her pyrexia. There was raised ESR, CRP and serum ferritin, with negative RA factor, ANTI CCP and ANCA. Her liver and renal function tests were normal. Her random blood sugar was $96 \mathrm{mg} / \mathrm{dl}$ with HbA1c of $5.6 \mathrm{gm} \%$. HIV serology was negative. Urine and Blood cultures were negative. ANA was +1 in the dilution of 1:100 with homogenous nucleolar pattern with negative ANA profile. Her bone marrow aspiration and biopsy were suggestive of reactive trilineage haematopoiesis and normocellular reactive marrow. Serum protein electrophoresis did not have any monoclonal bands and IGg4 levels were normal. Her 2-D echo was within normal limits and X-ray chest and that of the right shoulder joint did not detect any abnormality. Her CT scan of the abdomen and chest was suggestive of single reactive enlarged right axillary lymph nodes seen with ill-defined mildly enhancing soft tissue and fat stranding. Few fibrocalcific changes seen in both lung apices, suggestive of old healed granulomatous lesion.

When patient presented to us, she had fever with high suspicion of inflammatory arthropathy for which she underwent an MRI of right shoulder joint and a synovial fluid aspiration and analysis. Her MRI showed mild irregularity of acromion-clavicular joint, peripheral hypertrophy, osteophytes and moderate effusion in subacromial bursa (Figures 1 to 4). The synovial fluid aspiration showed total leucocyte count of 8000 cells $/ \mathrm{mm}^{3}$ with $72 \%$ neutrophils, $23 \%$ lymphocytes, $5 \%$ macrophage, protein of $4.4 \mathrm{gm} / \mathrm{dl}$, sugar of $40 \mathrm{mg} / \mathrm{dl}$ and no bacteria or AFB on gram stain or ZN stain. There were no organisms grown on the culture from the fluid. Cartridge based nucleic acid amplification test (CBNAAT) of the synovial fluid detected mycobacterium tuberculosis without rifampicin resistance.

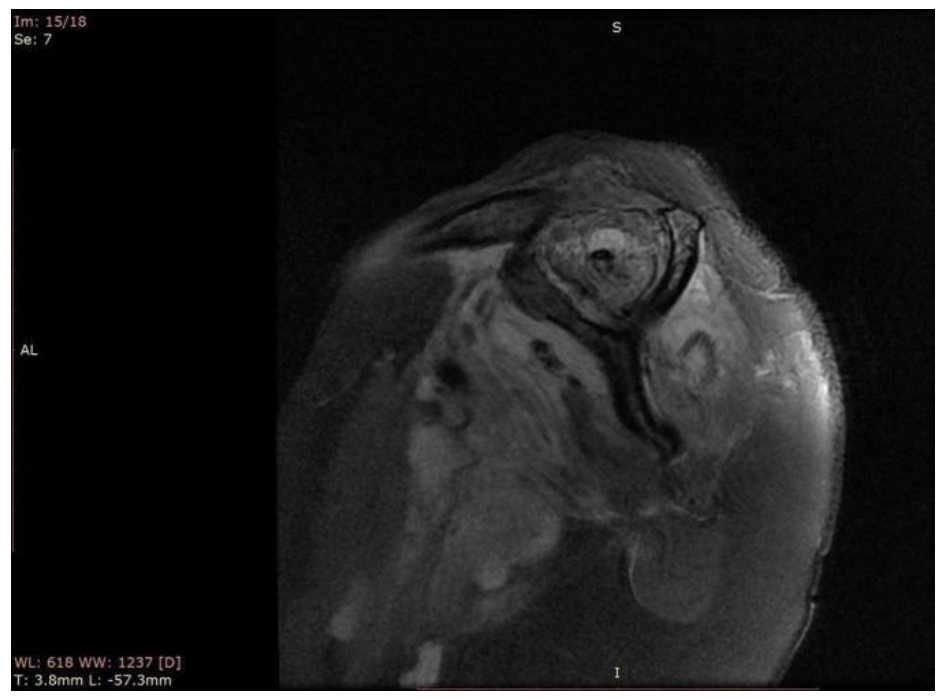

Figure 1: Decreased muscle bulk with fatty infiltration in muscles of rotator cuff 


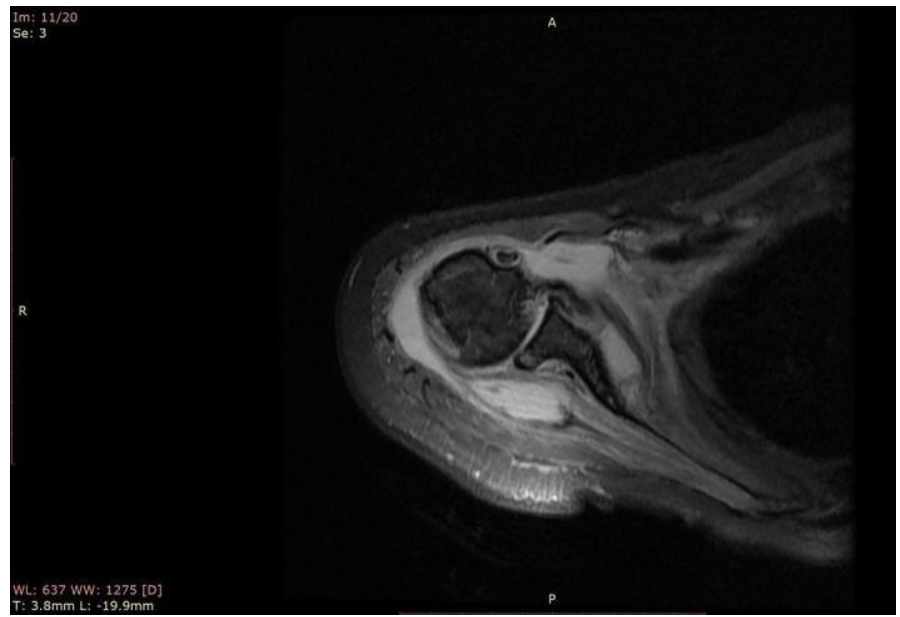

Figure 2: Erosion in peripheral humerus and posterior glenoid with joint effusion

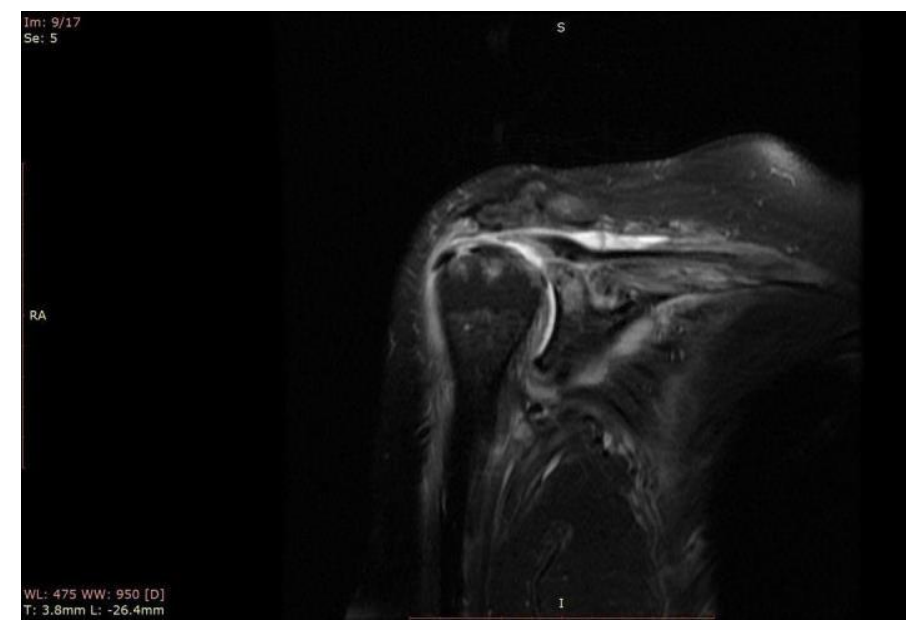

Figure 3: Superior subluxation of head of humerus with tear in supraspinatus with marrow oedema in glenoid fossa

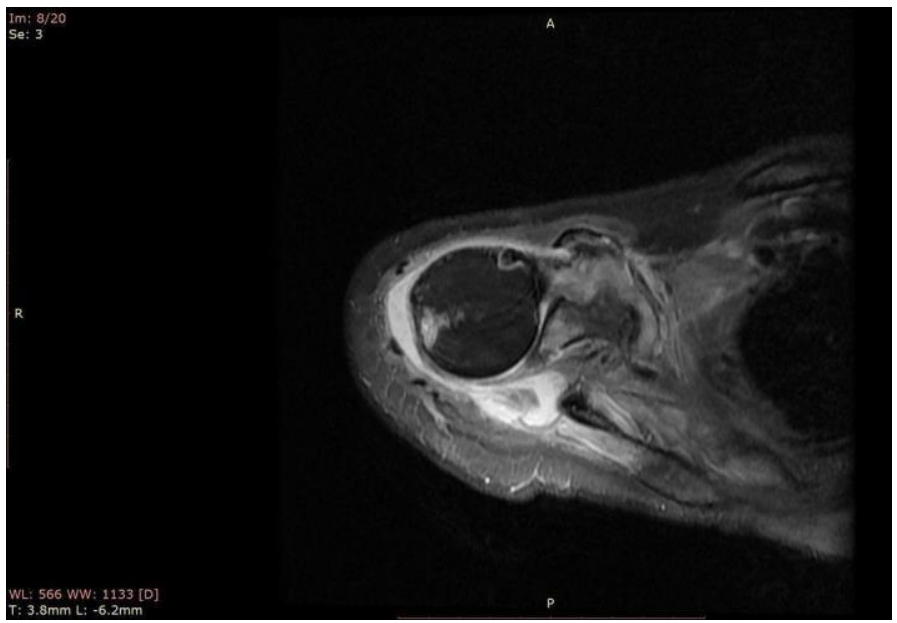

Figure 4: Synovial thickening

As per updated criteria of PUO, she can be considered for differential diagnosis of this syndrome because she had fever which was higher than $38 .{ }^{\circ} \mathrm{C}$ on several occasions, duration of fever was of more than three weeks and cause of fever was not certain after one week of the study in the hospital ${ }^{7}$. On initial presentation fever was without any localizing sign and tests for tropical infections like malaria, dengue, typhoid, leptospira, urinary tract infection was 
negative. The only useful pointer was a "frozen shoulder" of fifteen days duration. Adhesive capsulitis, relatively common condition, especially in females could be a mistaken diagnosis in this patient as her predominant complaints were related to fever. Infections like bacterial, viral, mycobacterial and fungal, Lyme's disease, trauma, crystal induced arthritis can be cause of mono-arthropathy ${ }^{8}$. Many of causes mentioned for monoarthritis affects bigger joints especially knee joint, however the common aphorism that acute monoarthritis unless proved otherwise can be considered due to infections was applied in this case. In this patient septic arthritis giving rise to fever was a possibility. Conditions leading to polyarthritis may present initially with monoarthritis and can be considered for differential diagnosis. Investigations especially synovial fluid aspiration with CBNAAT clinched diagnosis of tubercular affection of shoulder joint giving rise to fever.

Patient was given empiric treatment with third generation cephalosporin and NSAIDS on first admission. She had to be readmitted because of her pyrexia and on second admission, she received fluoroquinolones and glucocorticoids. These had given her partial relief. On admission with us, diagnosis of tubercular arthritis was made and was started on anti-tubercular drugs with daily regime of Isoniazid, Rifampicin, Pyrazinamide and Ethambutol (HRZE) 3 tablets per day.

Patient's condition improved after the treatment. There was no episode of fever. On follow up after 15 days the patient was free from fever, pain got reduced and she was able to do some daily activities with her right shoulder also.

\section{DISCUSSION:}

In 1961, Petersdrof and Beeson first define PUO as "Fever lasting for more than 3 weeks with temperature more than 38.3 degree Celsius on several occasions and no diagnosis after one week of indoor investigations"9. In 1991, Durack and Street proposed a new classification of pyrexia of unknown origin: "1. Classic PUO, 2. Nosocomial PUO. 3. Neutropenic PUO 4. HIV associated PUO" 10 Our case was fitting in the definition of PUO. The single most common infectious cause of PUO in developing countries is extrapulmonary tuberculosis or miliary tuberculosis ${ }^{11}$. There are certain points which help us point towards the diagnosis of tuberculosis in a patient of PUO like, organ-based symptoms or signs, past history of tuberculosis, history of contact with smear positive patients, subtle lung infiltrates on previous $\mathrm{X}$-rays, malnutrition, immunosuppression, diabetes, chronic renal insufficiency, patient on haemodialysis or renal transplant, homeless patient or patient living with HIV/AIDS (PLHA) ${ }^{12}$. $\mathrm{X}$ raychest of this patient had some changes which can be correlated with her pyrexia and tubercular arthritis. 
Skeletal tuberculosis is a rare disease, accounting for $10-35 \%$ of the extra pulmonary tuberculosis ${ }^{12-16}$. The most common form of skeletal tuberculosis found is pott's disease, which accounts for half of the musculoskeletal tuberculosis cases. The next is tubercular arthritis followed by extraspinal tubercular osteomyelitis ${ }^{14}$ In developing countries, musculoskeletal tuberculosis usually manifests clinically in the year following primary lung infection ${ }^{15}$. Tubercular arthritis can involve any joint, but is more common in hip and knee joints and is usually monoarticular. However, $10-15 \%$ can have polyarticular involvement ${ }^{12-16}$

Tuberculosis of the shoulder joint is extremely rare, the incidence is $0.9-1.7 \%$ of skeletal tuberculosis ${ }^{6,12,16}$. Tuberculosis of the shoulder joint can be classified as three types 6 Type 1: "caries sicca", the dry form, where the patient has wasting of the shoulder and restriction throughout the range of movements. Type 2: "caries exudate", fulminant form, here the patient has cold abscess and/or sinus formation. Type 3: "caries mobile" here the patient has restriction of movement only during active movements, and passive movements are normal 6. Our patient did not have swelling, cold abscess and/or sinus formation thus can be labelled as type 1, classical adult variety, that is, "caries sicca" type. In initial stage, our patient maybe in "caries mobile" stage which did not receive due attention and when she came to us in "caries sicca" stage, there was restriction of movement and her MRI and synovial fluid CBNAAT could confirm the diagnosis.

Patel PR et al, from Ahmedabad, Gujarat, India studied 306 patients of osteoarticular tuberculosis in between January 1989 to June 1999 of which 12 (9 males and 3 females, 11 adults, one child) had tuberculosis of shoulder joint; $50 \%$ of whom were misdiagnosed as having frozen shoulder. Outcome was good in their patients with anti-tubercular therapy. One case needed arthrodesis. Three patients regained full movements; others had mild restriction of movements ${ }^{6}$.

MRI is an important diagnostic tool for the early diagnosis of tubercular arthropathy and its differentiation from osteomyelitis. MRI being accurate in the prediction of severity and being noninvasive it is more often than not used as the preferred modality of early diagnosis. Mittal $\mathrm{R}$ et al. concluded that even though the radiography suggests of lytic lesions with osteoporosis, confirmation of tubercular arthritis can only be possible via histological or bacteriological evidence ${ }^{17}$. The tissue can be obtained by needle aspiration and/or biopsy, diagnosis can also be obtained by synovial fluid examination or synovial biopsy. However, the synovial fluid examination is often nonspecific. The synovial fluid can be subjected to Ziehl-Neelsen (ZN) staining, TB culture and TB PCR. PCR is a method with high sensitivity (73.0\%) and specificity (93.7\%), its sensitivity is higher in spinal samples (90\%). It also has a low false positivity $(13.6 \%)^{18}$. Our patient was subjected to a synovial fluid aspiration followed by analysis in which the routine, microscopy of the synovial fluid was normal with no staining for 
$\mathrm{ZN}$ stain and no organism grown on the culture media. The patient's synovial fluid was subjected to PCR which was positive for mycobacterium tuberculosis.

\section{CONCLUSION:}

Extra pulmonary tuberculosis can be one of the common causes of patient presenting with PUO; tuberculosis of the shoulder joint is even rarer. Musculoskeletal tuberculosis can be primary an isolated lesion, without active tuberculosis. This case demonstrates that how clinical suspicion, appropriate imaging and tissue examination with CBNAAT may result in diagnosis of extra pulmonary tuberculosis and ultimately a good outcome for the patient.

\section{REFERENCES:}

1. Bofinger JJ, Schlossberg D. Fever of unknown origin caused by tuberculosis. Infect Dis Clin North Am. 2007 Dec;21(4):947-62.

2. Attard L, Tadolini M, De Rose DU, Cattalini M. Overview of fever of unknown origin in adult and paediatric patients. Clin Exp Rheumatol. 2018;110: 10-24.

3. Pang Y, An J, Shu W, et al. Epidemiology of Extra pulmonary Tuberculosis among Inpatients, China, 2008-2017. Emerg Infect Dis. 2019;25(3):457-464.

4. Garg RK, Somvanshi DS. Spinal tuberculosis: a review. J Spinal Cord Med. 2011;34(5):440-454.

5. Gupta N, Bhatnagar AK. Musculoskeletal manifestations of tuberculosis: An observational study. J Family Med Prim Care. 2018;7(3):538-541

6. Patel P R, Patel D A, Thakker T, Shah K, Shah V B. Tuberculosis of shoulder joint. Indian J Orthop 2003;37: Bofinger JJ, Schlossberg D. Fever of unknown origin caused by tuberculosis. Infect Dis Clin North Am. 2007 Dec;21(4):947-62.

7. Roth AR and Basello GM. Approach to the adult patient with Fever of Unknown Origin. Am Fam Phys 2003; 68:2223-29

8. Sack K.Monarthritis: differential diagnosis. Am J Med. 1997;102(1A):30S

9. Petersdorf RG, Beeson PB. Fever of unexplained origin: report on 100 cases. Medicine (Baltimore) 1961; 40:1-30.

10. Durack DT, Street AC. Fever of unknown origin - reexamined and redefined. Curr Clin Top Infect Dis. 1991;11:35-51

11. Haq SA, Alam MN, Hossain SM, Dhar UK, Rahim S, Rahman M, et al. A study of prolonged pyrexia in Dhaka. Bangladesh Med Res Counc Bull 1996; 22:33-42.

12. Sharma SK, Mohan A. Extrapulmonary tuberculosis. Indian J Med Res 2004:120:316.

13. Vohra R, Kang HS, Dogra S, et al. tuberculosis osteomyelitis. J Bone Joint Surg Br 1997:79;562.

14. Held MFG, Hoppe S, Laubscher M et al. Epidemiology of musculoskeletal tuberculosis 
in a area with high disease prevalence Asian Spine J 2017:11;405.

15. Kumar K, Saxena MB. Multifocal osteoarticular tuberculosis. Int Orthop 1998:12;315.

16. Ostrowska M, Gietka J, Nesteruk T, Piliszek A, Walecki J. Shoulder joint tuberculosis.

17. Pol J Radiol. 2012;77(4):55-59.

18. Mittal R, Kandwal P, Rao SS, Kumar R. Acromioclavicular joint tuberculosis: apropos of two cases. Acta OrthopTraumatolTurc. 2014;48(5):590-2.

19. Pandey V, Chawla K, Acharya K. role of polymerase chain reaction in management of osteoarticular tuberculosis. Int Orthop 2009 Jun:33(3);801-805.

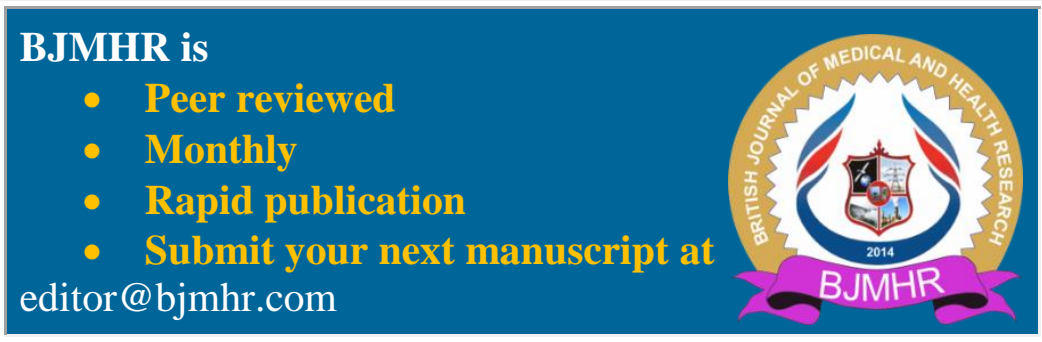

